\title{
Tei Index versus Pro BNP as Predictors of Weaning Failure in Mechanically Ventilated Chronic Obstructive Pulmonary Disease Patients
}

\author{
Hesham M. El-Ashmawy1, Tamer A. Helmy², Samier M. El-Awady², Doaa Hashad³, \\ Ahmed M. Nabil² \\ ${ }^{1}$ Department of Cardiology, Faculty of Medicine, Alexandria University, Alexandria, Egypt \\ ${ }^{2}$ Department of Critical Care Medicine, Faculty of Medicine, Alexandria University, Alexandria, Egypt \\ ${ }^{3}$ Department of Clinical Pathology, Faculty of Medicine, Alexandria University, Alexandria, Egypt \\ Email: ahmednabil571@yahoo.com
}

Received 4 June 2015; accepted 26 July 2015; published 29 July 2015

Copyright (C) 2015 by authors and Scientific Research Publishing Inc.

This work is licensed under the Creative Commons Attribution International License (CC BY).

http://creativecommons.org/licenses/by/4.0/

(c) (i) Open Access

\section{Abstract}

Objective: This prospective cohort study was designed to assess the rule of myocardial performance (Tei) index and NT-Pro BNP serum levels in prediction of weaning failure in mechanically ventilated COPD patients. Methods: Forty patients with respiratory failure due to acute exacerbation of COPD were enrolled to this study. All patients underwent a comprehensive echocardiographic examination including tissue doppler imaging within first 12 hours of admission. NT-pro BNP serum levels were measured on admission. Patients were followed up for outcome of weaning failure. Results: Right ventricular Tei index was higher in patients with failed weaning in comparison to successfully weaned patients (mean 0.66 versus $0.36, P<0.001$ ), also left ventricular Tei index was higher in patients who failed to be weaned (mean 0.45 versus $0.36, P=0.007$ ). There was no difference in serum NT-Pro BNP between successfully weaned patients and patients with weaning failure. Right ventricular Tei index showed higher accuracy in predicting weaning failure than the left ventricular Tei index (AUC 0.932 versus 0.754 ), while serum NT-Pro BNP showed no discrimination in predicting weaning failure (AUC 0.556). Conclusion: Tei index was superior to NT-ProBNP in prediction of weaning failure in COPD patients.

\section{Keywords}

Acute Exacerbation of COPD, Tei Index, Tissue Doppler Imaging, Pro BNP, Weaning, Mechanical Ventilation 


\section{Introduction}

COPD is still a burden and a major cause of mechanical ventilation worldwide. Weaning of COPD patients from mechanical ventilation (MV) is still a challenging process. Weaning means gradual or abrupt discontinuation of $\mathrm{MV}$; this decision depends on reversal of the cause of respiratory failure but extubation depends on conscious level and protection of airway. Spontaneous breathing trial is still a method that can test the ability of the patient to be weaned. It can be done with T piece trial or with pressure support at low level for 30 minutes up to 2 hours. The success depends on respiratory and cardiovascular compensations. Patients could be classified according to weaning fate into simple, difficult and prolonged weaning according to recommendations of international consensus conference sponsored by five major scientific societies that was held in Budapest, Hungary at 2005. Noninvasive positive pressure ventilation can assist in weaning in conscious cooperative patient with no copious secretions [1].

COPD is still considered systemic disease with multiple organ affections including cardiovascular affections with high incidence of right side of heart affections and also diastolic dysfunctions. This disorder affects weaning of the patients so weaning respiratory parameters alone usually fail to predict weaning success because they do not take into consideration cardiac reserve so during weaning process respiratory and cardiovascular reserve to be considered [2].

The myocardial performance (Tei) index is a reliable parameter suggesting global left and right ventricular function. Tei index has particularly been used for the evaluation of left ventricular function [3]. It has also been used to evaluate the right ventricular function in various pulmonary diseases such as pulmonary hypertension, COPD and cardiac amyloidosis. However, the evaluation of RV function by echocardiography has some limitations because of its geometry and complex contraction mechanism. Tissue Doppler imaging (TDI) echocardiography has been used to evaluate of the right ventricular function as a more quantitative method [4].

Pro BNP is good marker of myocardial strain. The role of increased Pro BNP level in risk stratification of AECOPD was documented in many studies [5] [6]. However, the relationship between Pro BNP and RV, LV Tie index in AECOPD has not been well evaluated yet. Therefore, in this study, we aimed to evaluate the relationship between the LV, RV Tei index and Pro BNP and compare between as predictors of difficult weaning.

\section{Methods}

\subsection{Study Design and Participants}

This Prospective cohort enrolled forty mechanically ventilated patients according to sample size calculation with AECOPD. The study was conducted at a university hospital, a tertiary care hospital at critical care department, the study was approved by the local ethics committee and written informed consent was obtained from all patients.

Patients with Chest trauma. Prior cardiothoracic surgery, hemodynamic instability, patients compliant to noninvasive mechanical ventilation and or those technically difficult transthoracic echocardiographic techniques were excluded from the study. Hemodynamic instability was defined by the presence a systolic blood pressure < 90 or need of vasopressor therapy. Patients were followed up till discharge from ICU.

\subsection{Variables}

The baseline characteristics of all patients including age, sex, vital signs, smoking index, clinical symptoms, other co morbidities, APACHE II and CAPS score were recorded carefully. Biochemical blood tests (arterial blood gases, NT-Pro BNP) were obtained from the entire study population on admission.

\subsection{Diagnosis and Treatment of AECOPD}

Previous pulmonary function test was present in most of the patients, only five patients we depend on history, clinical and radiological findings. Standard protocol of treatment according to GOLD 2014 recommendations with ventilator management, antibiotic, steroid, nebulizer, prophylactic low-molecular-weight heparin.

\subsection{Echocardiographic Study}

All study population underwent transthoracic echocardiographic evaluation including 2-dimensional (2D), M- 
mode, pulse wave Doppler imaging and TDI according to the recommendations of the American Society of Echocardiography using a commercially available system (Vivid 3, GE Vingmed Ultrasound AS, Horten, Norway). Echocardiographic assessment was performed within the first 12 hours. Subjects were examined in recumbent position. Tei index was calculated from recordings of five consecutive cardiac cycles with simultaneous electrocardiography using tissue Doppler at medial and lateral mitral and tricuspid annulus then the mean were calculated. Firstly, the time intervals; (a) from the end to the onset of tricuspid annular velocity pattern and the duration of the myocardial systolic wave (Sm), (b) from the onset to the end of the Sm was measured from the TDI recordings. Then, Tei index was computed by using the following equation: [isovolumetric contraction time (IVCT) + isovolumic relaxation time (IVRT)]/ejection time $=\mathrm{a}-\mathrm{b} / \mathrm{b}$ (Figure 1). RV Tei index normal range $0.28 \pm 0.04$ and of left $0.37 \pm 0.05$. All echocardiographic examination was performed by the same physician who was blinded to the results of the patient's data.

\subsection{Biochemical Analysis}

NT-Pro BNP was done on admission $2 \mathrm{~cm}$ of venous blood were taken at EDTA tube. The sample can be stable at room temperature for 72 hours. The samples were centrifuged and plasmas were then analyzed within $2 \mathrm{~h}$ using a monoclonal electro-chemiluminescence immunoassay, performed on a Roche Elecsys 2010 automated platform (Roche Diagnostics, Basel, Switzerland). The assay has an effective measuring range of 5 - 35,000 $\mathrm{ng} / \mathrm{L}$.

\subsection{Trial Weaning and Grouping}

According to recommendation of international consensus conference T-piece trial was initiated after 48hours to all studied patients. patients were categorized into two groups according to the fate of this trial group 1 who were succeeded T-piece trial with readiness to wean and group 2 who failed T-piece trial returned back to vetilatory support comparison between the two groups as regard age, sex, smoking index, APACHE II score, CAPS score, Tei index for the right and left ventricles and Pro BNP.

\subsection{Statistical Analysis}

Data were fed to the computer and analyzed using IBM SPSS software package version 20.0. Qualitative data were described using number and percent. Quantitative data were described using mean and standard deviation for normally distributed data while abnormally distributed data was expressed using median, minimum and maximum. Comparison between different groups regarding categorical variables was tested using Chi-square test. For normally distributed data, comparison between two independent populations were done using independent t-test. For abnormally distributed data, comparison between two independent populations were done using Mann Whitney test. Agreement of the different predictives with the outcome was used and was expressed in sensitivity, specificity, positive predictive value, negative predictive value and accuracy. Receiver operating characteristic curve (ROC) was plotted to analyze a recommended cutoff, the area under the ROC curve denotes the diagnostic performance of the test. Area more than $50 \%$ gives acceptable performance and area about $100 \%$ is the best performance for the test. Significance of the obtained results was judged at the $5 \%$ level.

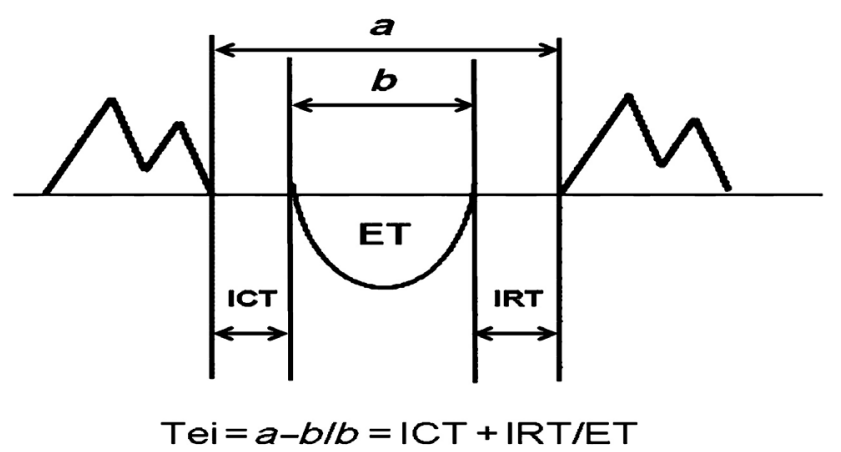

Figure 1. Tei index was calculated as: [isovolumetric contraction time (ICT) + isovulemic relaxation time (IRT)]/RV ejection time (ET) [3]. 


\section{Results}

\subsection{Patient Characteristics}

In the present study 38 (95\%) patients presented to the emergency department and 2 (5\%) patients were diagnosed during hospitalization. There was no difference between two groups as regard age sex and smoking index. The age of patients of group 1 ranged from 45 to 73 with a mean of $62.60 \pm 7.03$ years and the age of patients of group 2 ranged from 45 to 73 with a mean of $62.60 \pm 7.03$ years there was insignificant correlation between age and weaning failure. Group 1 included only one female patient (5\%) and rest of patients were males 15 (95\%) and group 2 included also one female patient and rest of patients were males 23 (95\%). the mean value of smoking index of group 1 was $52.12 \pm 5.53$ pack year and of group 2 was $51.44 \pm 5.48$ pack year. There was insignificant correlation between smoking index and weaning failure ( $P$ value 0.701$)$ (Table 1).

\subsection{Scoring Systems}

The mean value of APACHE II score of group 1 was $27.42 \pm 5.03$ and of group 2 was $27.25 \pm 4.30$. There was insignificant correlation between APACHE II score and weaning failure ( $P$ value 0.914$)$. The mean value of CAPS score of group 1 was $28.88 \pm 6.99$ and of group 2 was $28.06 \pm 7.31$. There was insignificant correlation between CAPS score and weaning failure ( $P$ value 0.726 ) (Table 1$)$.

\subsection{NT-Pro BNP, Tie Index and Weaning}

Pro BNP of group 1 ranged from 650.0 to 5000.0 with a mean of $2875.0 \mathrm{ng} / \mathrm{l}$. Pro BNP of group 2 ranged from 900.0 to 5200.0 with a mean of $2600.0 \mathrm{ng} / \mathrm{l}$. there was insignificant correlation between Pro BNP and weaning failure ( $P$ value 0.553 ) but pro BNP at level of $3900 \mathrm{ng} / \mathrm{L}$ predict failure of weaning with a sensitivity of $37.5 \%$ and specificity of $87.5 \%$ (area under the curve, 0.556 ; $P$ 0.553). Positive predictive value was $66.67 \%$ and negative predictive value was $67.74 \%$ and accuracy of 67.5 (Table 2, Figure 2).

Table 1. Relation between weaning and different parameters.

\begin{tabular}{cccc}
\hline & & Weaning & \\
\cline { 2 - 4 } & Weaned $(\mathrm{n}=24)$ & Failed $(\mathrm{n}=16)$ & \\
\hline Age & $60.96 \pm 6.48$ & $65.06 \pm 7.30$ & \\
Sex & & & \\
Male & $15(93.8 \%)$ & $23(95.8 \%)$ & $1(4.2 \%)$ \\
Female & $1(6.3 \%)$ & $52.12 \pm 5.53$ & 1.000 \\
Smoking index & $51.44 \pm 5.48$ & $27.42 \pm 5.03$ & 0.701 \\
APACHE II & $27.25 \pm 4.30$ & $0.36(0.27-0.48)$ & 0.914 \\
CAPS score & $28.06 \pm 7.31$ & $0.36(0.32-0.49)$ & 0.726 \\
Tei index right & $0.66(0.39-0.90)$ & $2875.0(650.0-5000.0)$ & $0.007^{*}$ \\
Tei index left & $0.45(0.32-0.85)$ & & 0.553 \\
Pro BNP & $2600.0(900.0-5200.0)$ & & \\
\hline
\end{tabular}

* Statistically significant at $P \leq 0.05$.

Table 2. Agreement of Pro BNP, tei index right and left with weaning.

\begin{tabular}{|c|c|c|c|c|c|c|c|c|c|c|}
\hline & & \multicolumn{2}{|c|}{ Weaning } & \multirow{2}{*}{ AUC } & \multirow{2}{*}{$P$} & \multirow{2}{*}{ Sensitivity } & \multirow{2}{*}{ Specificity } & \multirow{2}{*}{ PPV } & \multirow{2}{*}{ NPV } & \multirow{2}{*}{ Accuracy } \\
\hline & & Successful & Failed & & & & & & & \\
\hline \multirow{2}{*}{ Pro BNP } & $\leq 3900$ & 21 & 10 & \multirow{2}{*}{0.556} & \multirow{2}{*}{0.553} & \multirow{2}{*}{37.50} & \multirow{2}{*}{87.50} & \multirow{2}{*}{66.67} & \multirow{2}{*}{67.74} & \multirow{2}{*}{67.50} \\
\hline & $>3900$ & 3 & 6 & & & & & & & \\
\hline \multirow{2}{*}{ Tei index right } & $\leq 0.43$ & 21 & 2 & \multirow{2}{*}{$0.932^{*}$} & \multirow{2}{*}{$<0.001$} & \multirow{2}{*}{87.50} & \multirow{2}{*}{87.50} & \multirow{2}{*}{82.35} & \multirow{2}{*}{91.30} & \multirow{2}{*}{87.50} \\
\hline & $>0.43$ & 3 & 14 & & & & & & & \\
\hline \multirow{2}{*}{ Tei index left } & $\leq 0.41$ & 23 & 8 & \multirow{2}{*}{$0.754^{*}$} & \multirow{2}{*}{0.007} & \multirow{2}{*}{50.0} & \multirow{2}{*}{95.83} & \multirow{2}{*}{88.89} & \multirow{2}{*}{74.19} & \multirow{2}{*}{77.50} \\
\hline & $>0.41$ & 1 & 8 & & & & & & & \\
\hline
\end{tabular}




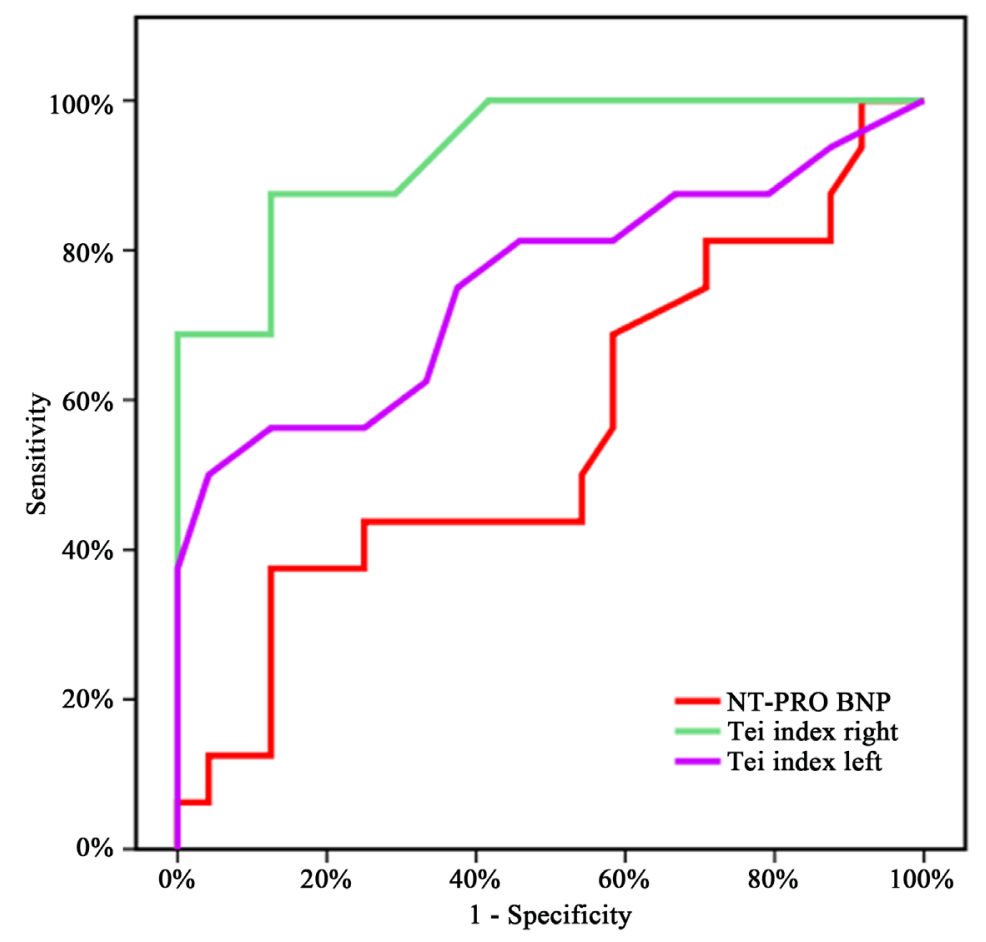

Figure 2. ROC curve for Pro BNP, Tei index right and left for predicting weaning failure.

Tei index of right ventricle of group 1 ranged from 0.27 to 0.48 with a mean 0.66 . Tei index of right ventricle of group 2 ranged from 0.39 to 0.90 with a mean of 0.66 . There was significant correlation between Tei index of right ventricle and weaning failure $P$ value $<0.001^{*}$. Tei index of right ventricle at level of 0.43 predict failure of weaning with a sensitivity of $87.5 \%$ and specificity of $87.5 \%$ (area under the curve, $0.932 ; P<0.001$ ). Positive predictive value was $82.35 \%$ and negative predictive value was $91.30 \%$ and accuracy of 87.5 (Table 2, Figure 2).

Tei index of left ventricle of group 1 ranged from 0.32 to 0.49 with a mean of 0.36 . Tei index of left ventricle of group 2 ranged from 0.32 to 0.85 with a mean of 0.45 . There was significant correlation between Tei index of left ventricle and weaning failure $\left(P\right.$ value $\left.0.007^{*}\right)$. Tei index left at level of 0.41 predict failure of weaning with a sensitivity of $95.83 \%$ and specificity of $95.83 \%$ (area under the curve $0.754 ; P 0.007$ ). Positive predictive value was $88.89 \%$ and negative predictive value was $74.19 \%$ and accuracy of 77.5 (Table 2, Figure 2).

\section{Discussion}

Multiple studies have addressed the question of whether BNP or NT-pro-BNP could be used to identify patients who fail to wean for cardiac reasons [7]-[14]. The study by Zapata et al. was a prospective observational study of 100MV patients [15]. All patients underwent spontaneous breathing trials over 48 hours and were assessed by transthoracic echocardiography, pulmonary artery catheter and BNP and NT-pro-BNP. They concluded that B-type natriuretic peptides, particularly BNP, can predict weaning failure due to heart failure (HF) before an SBT. Increases in natriuretic peptides during SBT are diagnostic of HF as the cause of weaning failure. BNP performs better than NT-pro-BNP in prediction and diagnosis of HF. The cut-off values using receiver operating (ROC) curve analyses to predict HF were $263 \mathrm{ng} / \mathrm{L}$ for BNP $(P<0.001)$ and $1343 \mathrm{ng} / \mathrm{L}$ for NT-pro-BNP $(P=$ 0.08). Mekontso-Dessap et al. [11] showed BNP levels after diuretic therapy were lower in patients with weaning success (517 pg/mL versus $226 \mathrm{pg} / \mathrm{mL}$ ). Grasso et al. [14] used N-terminal pro-BNP to detect acute cardiac dysfunction during weaning failure in difficult-to-wean patients with chronic obstructive pulmonary disease. He showed that plasma levels of NT-pro-BNP increased significantly at the end of the spontaneous breathing trial only in patients with acute cardiac dysfunction (median 12,733, interquartile range 16,456 pg/mL, $P<0.05$ ). Chien et al. [10] used the median BNP levels after the 2 hr SBT showing BNP levels were 461 (168 - 1202) 
$\mathrm{pg} / \mathrm{mL}, 418(218$ - 1085) pg/mL, and $224(112$ - 660) pg/mL in the SBT failure, extubation failure, and extubation success groups, respectively. Gerbaud et al. [12] prospectively evaluated 44 patients with echocardiography and NT-pro-BNP. NT-pro-BNP levels (8199 (3106 - 10949) versus 4200 (1855 - 7125) pg/mL, P=0.004) increased significantly in those who failed the SBT but in present study NT-pro BNP did not predict weaning failure significally but at level of higher than $3900 \mathrm{ng} / \mathrm{l}$ showed high specificity in prediction of failure of weaning this may be due to all enrolled patients were COPD patients with high right ventricular strain.

There is growing indication to advocate that transthoracic echocardiography (TTE) should be used to categorize the cardiac origin of respiratory weaning failure. The study by Gerbaud et al. looks at the weaning trials in congestive heart failure patient by analysis of the mitral Doppler inflow $E$ velocity to annular tissue Doppler $E$ a wave velocity $(E / E a)$ ratio measurement. Even though he concluded that TTE could not predict the outcome of SBT, he noticed cardiac index increased significantly at end-SBT in patients who passed [12]. In contrast, the study by Moschietto et al. in 68 patients on MV over 48 hours proved that measurement of the $E / E$ a ratio with TTE could predict weaning failure. Diastolic dysfunction with relaxation impairment was strongly associated with weaning failure. Additionally, the impossibility of enhancing the left ventricle relaxation rate during the SBT seemed to be the key factor of weaning failure. In contrast, the systolic dysfunction was not associated with weaning outcome [16]. Papanikolaou et al. evaluated 50 patients with Doppler echocardiography to predict outcome of weaning trials. The result indicated that LV diastolic dysfunction is significantly associated with weaning outcome in critically ill patients with preserved LV systolic function. An E/E $a$ ratio greater than 7.8 may identify patients at high risk of weaning failure [17]. Schifelbain et al. conducted randomized crossover clinical trial of 24 patients to analyze changes in cardiac function, using Doppler echocardiogram, in critical patients during weaning from MV. He used two different weaning methods: pressure support ventilation and T-tube. He did not find any differences between Doppler echocardiography and cardiorespiratory variables during pressure support ventilation and T-tube. However cardiac structures were smaller, isovolumic relaxation time was larger, and oxygenation level was greater in successfully weaned patients [18]. It is probably safe to say that Doppler echocardiography has a place for assessment of weaning failure due to cardiac origin if performed routinely in the ICU. However, due to certain limitation relating to patient, it cannot be used in every patient. In the present study we used Tei index or myocardial performance index which give clue of systolic and diastolic function to predict weaning failure. Many studies were done on relation between Tei index, mortality, right and left clinical and subclinical ventricular function, corpulmonale and pulmonary hypertension [19]-[24]. But to my knowledge no published studies till now on weaning in current study good correlation with weaning was found and superior to pro BNP.

\section{Study Limitations}

The present study has limitation, correlation between other biomarkers (e.g. brain natriuretic peptide (BNP), troponin and Tei index was not determined in this study.

\section{Conclusion}

This study showed that Tei index may be used as an easily repeated and quick method and superior to Pro BNP in the quantitative prediction of weaning failure.

\section{References}

[1] Boles, J.M., Bion, J., Connors, A., Herridge, M., Marsh, B., Melot, C., et al. (2007) Weaning from Mechanical Ventilation. European Respiratory Journal, 29, 1033-1056. http://dx.doi.org/10.1183/09031936.00010206

[2] Pinsky, M.R. (2000) Breathing as Exercise: The Cardiovascular Response to Weaning from Mechanical Ventilation. Intensive Care Medicine, 2, 1164-1166. http://dx.doi.org/10.1007/s001340000619

[3] Tei, C., Ling, L.H., Hodge, D.O., Bailey, K.R., Oh, J.K., Rodeheffer, R.J., et al. (1995) New Index of Combined Systolic and Diastolic Myocardial Performance: A Simple and Reproducible Measure of Cardiac Function-A Study in Normals and Dilated Cardiomyopathy. Journal of Cardiology, 26, 357-366.

[4] Tei, C., Dujardin, K.S., Hodge, D.O., Bailey, K.R., McGoon, M.D., Tajik, A.J., et al. (1996) Doppler Echocardiographic Index for Assessment of Global Right Ventricular Function. Journal of the American Society of Echocardiography, 9, 838-847. http://dx.doi.org/10.1016/S0894-7317(96)90476-9

[5] Buchner, S., Riegger, G. and Luchner, A. (2004) Clinical Utility of the Cardiac Markers BNP and NT-proBNP. Acta Medica Austriaca, 31, 144-151. 
[6] Morrison, L.K., Harrison, A., Krishnaswamy, P., Kazanegra, R., Clopton, P. and Maisel, A. (2002) Utility of a Rapid B-Natriuretic Peptide Assay in Differentiating Congestive Heart Failure from Lung Disease in Patients Presenting with Dyspnea. Journal of the American College of Cardiology, 39, 202-209. http://dx.doi.org/10.1016/S0735-1097(01)01744-2

[7] Pirracchio, R., Salem, R. and Mebazaa, A. (2009) Use of B-Type Natriuretic Peptide in Critically Ill Patients. Biomarkers in Medicine, 3, 541-547. http://dx.doi.org/10.2217/bmm.09.45

[8] Principi, T., Falzetti, G., Elisei, D., Donati, A. and Pelaia, P. (2009) Behavior of B-Type Natriuretic Peptide during Mechanical Ventilation and Spontaneous Breathing after Extubation. Minerva Anestesiologica, 75, 179-183.

[9] Abroug, F. and Ouanes-Besbes, L. (2008) Detection of Acute Heart Failure in Chronic Obstructive Pulmonary Disease Patients: Role of B-Type Natriuretic Peptide. Current Opinion in Critical Care, 14, 340-347. http://dx.doi.org/10.1097/MCC.0b013e3282faa304

[10] Chien, J.Y., Lin, M.S., Huang, Y.C., Chien, Y.F., Yu, C.J. and Yang, P.C. (2008) Changes in B-Type Natriuretic Peptide Improve Weaning Outcome Predicted by Spontaneous Breathing Trial. Critical Care Medicine, 36, 1421-1426. http://dx.doi.org/10.1097/CCM.0b013e31816f49ac

[11] Mekontso-Dessap, A., de Prost, N., Girou, E., Braconnier, F., Lemaire, F., Brun-Buisson, C., et al. (2006) B-Type Natriuretic Peptide and Weaning from Mechanical Ventilation. Intensive Care Medicine, 32, 1529-1536. http://dx.doi.org/10.1007/s00134-006-0339-7

[12] Gerbaud, E., Erickson, M., Grenouillet-Delacre, M., Beauvieux, M.C., Coste, P., Durrieu-Jais, C., et al. (2012) Echocardiographic Evaluation and N-Terminal Pro-Brain Natriuretic Peptide Measurement of Patients Hospitalized for Heart Failure during Weaning from Mechanical Ventilation. Minerva Anestesiologica, 78, 415-425.

[13] Mekontso-Dessap, A., Tual, L., Kirsch, M., D’Honneur, G., Loisance, D., Brochard, L., et al. (2006) B-Type Natriuretic Peptide to Assess Haemodynamic Status after Cardiac Surgery. British Journal of Anaesthesia, 97, 777-782. http://dx.doi.org/10.1093/bja/ael271

[14] Grasso, S., Leone, A., De Michele, M., Anaclerio, R., Cafarelli, A., Ancona, G., et al. (2007) Use of N-Terminal ProBrain Natriuretic Peptide to Detect Acute Cardiac Dysfunction during Weaning Failure in Difficult-to-Wean Patients with Chronic Obstructive Pulmonary Disease. Critical Care Medicine, 35, 96-105. http://dx.doi.org/10.1097/01.CCM.0000250391.89780.64

[15] Zapata, L., Vera, P., Roglan, A., Gich, I., Ordonez-Llanos, J. and Betbese, A.J. (2011) B-Type Natriuretic Peptides for Prediction and Diagnosis of Weaning Failure from Cardiac Origin. Intensive Care Medicine, 37, 477-485. http://dx.doi.org/10.1007/s00134-010-2101-4

[16] Moschietto, S., Doyen, D., Grech, L., Dellamonica, J., Hyvernat, H. and Bernardin, G. (2012) Transthoracic Echocardiography with Doppler Tissue Imaging Predicts Weaning Failure from Mechanical Ventilation: Evolution of the Left Ventricle Relaxation Rate during a Spontaneous Breathing Trial Is the Key Factor in Weaning Outcome. Critical Care, 16, R81. http://dx.doi.org/10.1186/cc11339

[17] Papanikolaou, J., Makris, D., Saranteas, T., Karakitsos, D., Zintzaras, E., Karabinis, A., et al. (2011) New Insights into Weaning from Mechanical Ventilation: Left Ventricular Diastolic Dysfunction Is a Key Player. Intensive Care Medicine, 37, 1976-1985. http://dx.doi.org/10.1007/s00134-011-2368-0

[18] Schifelbain, L.M., Vieira, S.R., Brauner, J.S., Pacheco, D.M. and Naujorks, A.A. (2011) Echocardiographic Evaluation during Weaning from Mechanical Ventilation. Clinics (Sao Paulo), 66, 107-111. http://dx.doi.org/10.1590/S1807-59322011000100019

[19] El-Damarawy, M., Zeidan, H. and Suwailem, S. (2008) Myocardial Performance Index in Patients with Chronic Obstructive Pulmonary Disease. Heart Mirror Journal, 2, 1687-1692.

[20] Tanaka, Y., Hino, M., Mizuno, K. and Gemma, A. (2013) Evaluation of Right Ventricular Function in Patients with COPD. Respiratory Care, 58, 816-823.

[21] Das, M., Tapadar, S.R., Mahapatra, A.B., Chowdhury, S.P. and Basu, S. (2014) Assessment of RV Function in Patients of (COPD). Journal of Clinical and Diagnostic Research, 8, 11-13. http://dx.doi.org/10.7860/jcdr/2014/6440.4090

[22] Sabit, R., Bolton, C.E., Fraser, A.G., Edwards, J.M., Edwards, P.H., Ionescu, A.A., et al. (2010) Sub-Clinical Left and Right Ventricular Dysfunction in Patients with COPD. Respiratory Medicine, 104, 1171-1178. http://dx.doi.org/10.1016/j.rmed.2010.01.020

[23] Sadic, B.O., Euryksel, E. and Tanrikulu, M.A. (2011) Right Ventricular Function in Patient with Acute Exacerbation of COPD. Respiratory Medicine, 109, 1123-1135.

[24] Botelho, C.M.A., Pena, J.L.B., Colosimo, E.A. and Moreira, M.C.V. (2008) B-Type Natriuretic Peptide (BNP) Regulation and Doppler Echocardiographic Alterations in Patients with Chronic Obstructive Pulmonary Disease Acute Exacerbations. European Journal of Heart Failure Supplements, 7, 82. http://dx.doi.org/10.1016/S1567-4215(08)60225-8 